\title{
Ageing of Polymer Frictional Interfaces: The Role of Quantity and Quality of Contact
}

\author{
D. Petrova, D. K. Sharma, M. Vacha, D. Bonn, A. M. Brouwer, and B. Weber* \\ Cite This: ACS Appl. Mater. Interfaces 2020, 12, 9890-9895 \\ Read Online
}

ABSTRACT: When two objects are in contact, the force necessary for one to start sliding over the other is larger than the force necessary to keep the sliding motion going. This difference between static and dynamic friction is thought to result from a reduction in the area of real contact upon the onset of slip. Here, we resolve the structure in the area of contact on the molecular scale by means of environment-sensitive molecular rotors using (super-resolution) fluorescence microscopy and fluorescence lifetime imaging. We demonstrate that the macroscopic friction force is not only controlled by the area of real contact but also controlled by the "quality" of that area of real contact, which
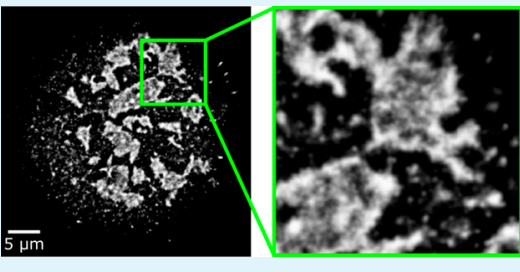
determines the friction per unit contact area. We show that the latter is affected by the local density of the contacting surfaces, a parameter that can be expected to change in time at any interface that involves glassy, amorphous materials.

KEYWORDS: frictional ageing, contact, fluorescence lifetime, super-resolution, polymer, glass, mechanics, microscopy

\section{INTRODUCTION}

At most solid-on-solid interfaces, the static friction force that resists the sliding motion of the bodies increases with the logarithm of contact time. ${ }^{1-7}$ When a macroscopic slip is initiated, the static friction force drops to its dynamic value. If the reduction in friction force with sliding distance is much sharper than that in the driving force, a stick-slip instability results. Unwanted stick-slip instabilities can cause destructive vibrations in, for example, earthquakes, ${ }^{8}$ articular joints, ${ }^{9}$ brakes, ${ }^{10}$ drilling of oil and gas, ${ }^{11}$ and boat propulsion systems. ${ }^{12}$ The reduction in friction at the onset of macroscopic sliding is often attributed to a reduction in the area of real contact $\mathrm{A}$ when the sliding starts. ${ }^{1}$ At rest, the contacts between the two surfaces age: plastic flow ${ }^{13,14}$ of the asperities cause a gradual increase in A. The area of real contact is however reduced again when the interface starts to slip because microcontacts that have aged are replaced by new, younger contacts. ${ }^{1}$ The friction force $F_{\mathrm{f}}$ is widely assumed to be proportional to the area of real contact. ${ }^{15,16}$ Therefore, as A is reduced, so is the friction force. ${ }^{17-19}$ Exceptions to this behavior have also been observed, notably by Bureau et al. ${ }^{20}$ who observed that polymer-on-glass interfaces display glassy behavior which involves a varying interfacial shear stress. More recently, lateral force microscopy experiments have demonstrated that the interfacial shear stress can also increase because of the formation of chemical bonds across a frictional interface during contact ageing. ${ }^{4,21}$ Furthermore, recent results for polymer-glass interfaces ${ }^{22}$ suggest that the proportionality between the macroscopic friction force and the microscopic area of real contact may not always hold; visualization of the contact area during slide-hold-slide experiments demonstrates that the increase in the static friction coefficient with ageing time is larger than that of the area of real contact: the friction force per unit contact area increases. ${ }^{23}$ The difference between static and dynamic friction is then not only a consequence of the increase in contact area but also an increase in the shear strength of the contacts. This behavior is in line with the earlier observation of strain-hardening plasticity of such polystyrene (PS)-on-glass contacts; the PS surface is plastically compacted upon contact with glass ${ }^{22,23}$ and hardens as it is strained. In other words, the PS becomes mechanically stronger as it is compacted in the asperities, which could also lead to the increased shear strength, as the sliding is accommodated by the yielding PS surface. ${ }^{22,23}$

Clearly, reliable experimental measurement of the area of real contact is of critical importance to distinguish between the size of the contact (area, measured in $\mathrm{m}^{2}$ ) and the quality of the contact (shear strength, measured in $\mathrm{Pa}$ ). In our previous work, the area of real contact was measured through diffraction-limited fluorescence intensity imaging. ${ }^{22,23}$ Here, we critically evaluate the precision of this type of contact area measurement by means of super-resolution microscopy ${ }^{24,25}$ and fluorescence lifetime imaging (FLIM). ${ }^{26}$

Our results indicate that diffraction-limited imaging is sufficient to resolve the PS-on-glass contacts. We also show that the strain-hardening behavior is observed not only at the PS-on-glass interfaces that are primarily studied here but also at other amorphous interfaces: the shear strength generated by

Received: October 27, 2019

Accepted: February 4, 2020

Published: February 6, 2020 


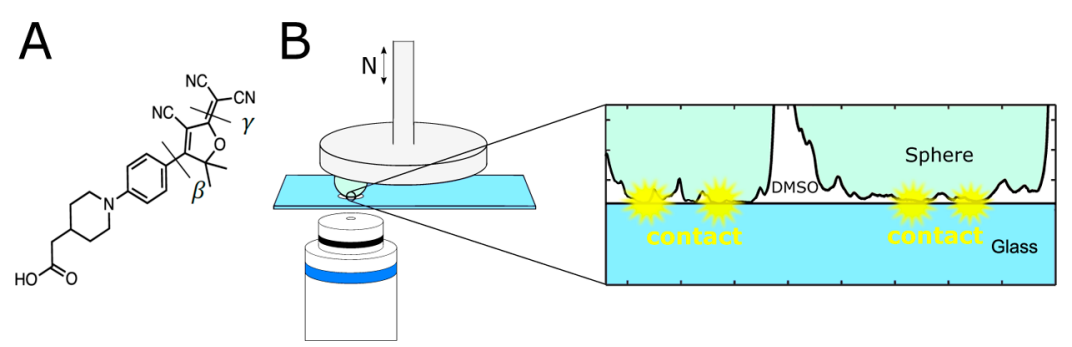

Figure 1. (A) Molecular structure of the DCDHF probe molecules. (B) Schematic presentation of the experiments in which a sphere is brought into contact with a glass coverslip with normal force $N$. The enhanced fluorescence of the probe molecules indicates contact. To suppress strong light scattering, contacts are immersed in formamide, a low viscosity liquid that does not affect the friction behavior. ${ }^{22,23}$ Figure recreated from ref 27.

poly(methyl methacrylate) (PMMA)-on-glass contacts also grows in time. We find that for both PS and PMMA-on-glass contacts, the interfacial polymer density increases in time when the interface is loaded, suggesting that densification of glassy polymer surfaces in contact may be a universal phenomenon. Other glassy polymers can also be expected to display contact ageing driven by an evolution of the shear strength or friction per unit contact area.

\section{EXPERIMENTAL SECTION}

For the friction and visualization experiments, a rheometer measuring head is mounted on top of a laser scanning confocal microscope, with a sphere glued to the rheometer tool (Figure 1). Lowering the rheometer tool creates an interface between the sphere and the glass coverslip that is loaded with an imposed normal force $N$. The rheometer tool can also be rotated, which allows the measurement of the friction force through the torque measurement on the rotation axis.

The fluorescence intensity of a viscosity-sensitive molecular rotor-2-(1-(4-(4-cyano-5-(dicyanomethylene)-2,2-dimethyl-2,5-dihydrofuran-3-yl)phenyl)-piperidin-4-yl) acetic acid (DCDHF) ${ }^{28,29}-$ is used as a local molecular environment sensor at the interface. A glass coverslip with a covalently attached monolayer of DCDHF is inserted into the inverted confocal microscopy setup. The fluorophores at the interface are excited-and their emission is detected-through the transparent substrate by the inverted microscope. At the solid-solid interface, fluorescence arises ${ }^{28,29}$ because the rotations around the $\beta$-bond or the $\gamma$-bond of the DCDHF molecules in the monolayer, which give rise to rapid nonradiative decay, are suppressed.

We perform slide-hold-slide friction experiments in which we first bring a rough PS sphere of $600 \mu \mathrm{m}$ diameter in contact with a smooth float glass substrate at a contact force of approximately $400 \mathrm{mN}$. It is important to note that in this rough-on-smooth contact geometry, during the slip the sphere will continuously touch the substrate with the same asperities. ${ }^{23}$ The static friction force is measured as a function of waiting time: for each slip event, the sphere is forced to slide over the glass for a total sliding distance of $12 \mu \mathrm{m}$. During and in between the friction measurements, the normal force is held fixed. A single slide-hold-slide series consists of up to 13 friction measurements, conducted at increasing waiting times which are measured relative to the moment at which the normal force was first applied. After the very first "run-in" friction measurement, the measured friction forces are reproducible, see Figure 2 (error bars indicate deviations between independent experiments). During the first measurement, there is a significant increase in the area of real contact which results from the fact that this is the first moment at which the interface is subjected to large shear stresses. These shear stresses, similar to the normal stress, induce an increase of the area of real contact in time; shear stresses are known to accelerate contact ageing due to plastic flow. ${ }^{20}$ The subsequent friction measurements show that the static friction force grows logarithmically with time, an observation that has been made in many frictional systems. ${ }^{1-7,30,31}$

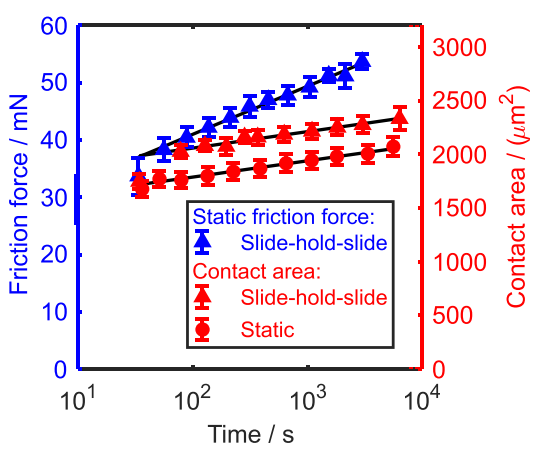

Figure 2. Ageing of the static friction force for PS on glass (in blue). All data sets show pronounced ageing: the static friction force increases logarithmically by almost a factor of 1.5 over the duration of the experiment. The black lines are semilogarithmic fits to the data. Red data points depict the growth of the PS-on-glass contact area with time as determined from molecular probe fluorescence images (see Figure 4) taken during a slide-hold-slide experiment and taken during a static ageing experiment, in which the contact is only loaded in the normal direction.

To understand what drives the logarithmic increase of the friction force with time, we performed atomic force microscopy measurements of the PS surface before and after contact with the smooth glass substrate. As shown in Figure 3, creep flow of the asperities of the PS occurs during the contact, causing the area of real contact between the sphere and the substrate to increase over time.

To quantify the contact area growth, we performed fluorescence experiments using our pressure-sensitive molecular probe. By applying an Otsu threshold on the fluorescence intensity images (see Figure 4), we plot the number of bright pixels (indicating real contact) multiplied with the pixel area as a function of waiting time in red in Figure 2. We find that the growth rate of the contact area in time is significantly smaller than that of the friction force; the creep growth in contact area is not strong enough to explain the observed increase in the static friction force. In other words, the quality of contact-or friction per unit contact area-must be increasing as the experiment progresses.

Critically, this conclusion only holds if the observed growth of the area of real contact is not affected by the limitation of the in-plane imaging resolution; the fluorescence observations of the area of real contact are molecularly sensitive in the axial direction but diffractionlimited [point spread function with a full width at half-maximum (fwhm) of $450 \mathrm{~nm}^{22}$ in the in-plane direction. To experimentally test the diffraction-limited observations, we apply super-resolution microscopy to the PS-on-glass contacts.

To image the exact same contact using a diffraction-limited and a super-resolved imaging technique, we first record a diffraction-limited fluorescence image of the aged contact immersed in dimethyl sulfoxide using widefield microscopy (Figure 5). After that, we expose the functionalized coverslip to intense laser light $\left(5 \mathrm{~kW} / \mathrm{cm}^{2}\right)$ for a few hours. In this way, we bleach the majority of the molecules 

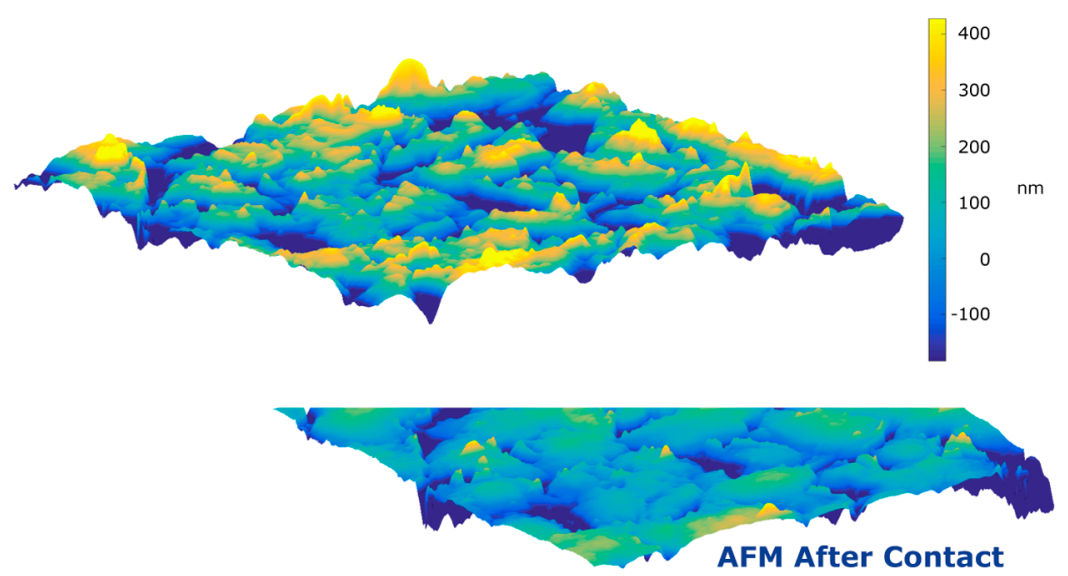

Figure 3. AFM images of the PS surface before and after contact with glass ( $400 \mathrm{mN}$ normal force for $30 \mathrm{~min}$ ). Comparison of the two images reveals that plastic flow of the asperities has occurred, resulting in a flattened overall topography. The AFM images were recorded at a pixel size of $32 \mathrm{~nm}$, larger than the scale at which convolution of the image with the tip radius is expected to impact the image. ${ }^{32}$

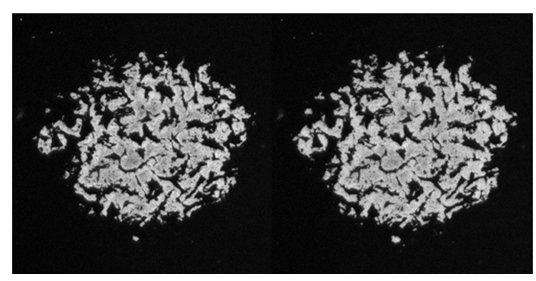

Figure 4. Molecular probe fluorescence images of a PS-on-glass contact after ageing for $30 \mathrm{~s}$ (left) and $3000 \mathrm{~s}$ (right, the same contact). An increase in contact area and coarsening of the contact structure due to the plastic flow in the asperities can be observed. Images are $90 \mu \mathrm{m} \times 90 \mu \mathrm{m}$.

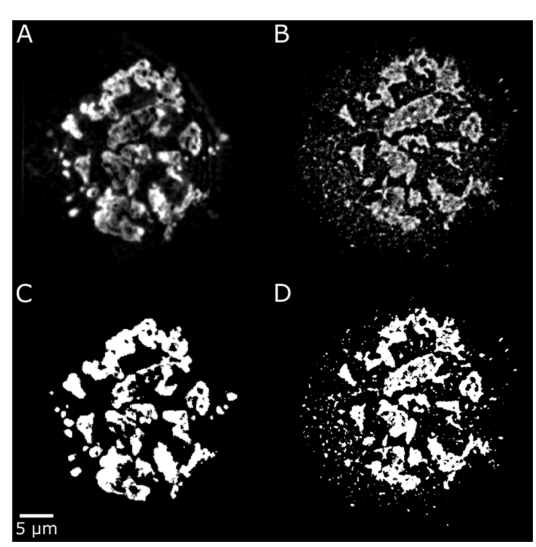

Figure 5. Area of real contact between a rough PS sphere and a smooth glass coverslip at a normal force of $30 \mathrm{mN}$ imaged using confinement-sensitive DCDHF molecules. (A) Area of real contact measured by diffraction-limited fluorescence imaging. (B) Area of real contact measured through super-resolution microscopy. (C) Uncertainty of the Otsu thresholding for diffraction-limited fluorescence imaging: by reducing the threshold value green contacts appear, by increasing the threshold value red contacts disappear. (D) Uncertainty of the contact area estimation for super-resolution microscopy images. By reducing the threshold value, green contacts appear, and by increasing the threshold value, red contacts disappear. To construct the super-resolved image, we represent each molecule that was located by a bright pixel and convolve the resulting image with the resolution ${ }^{36}$ (fwhm $152 \mathrm{~nm}$ ). The pixel size is $20 \mathrm{~nm}$. The images were recorded approximately 1 and $4 \mathrm{~h}$ after contact formation. and only a few fluorescent single molecules remain within the observed area during a single time frame of $100 \mathrm{~ms}$. Many of the molecules that were photobleached, however, spontaneously recover and switch several times between a fluorescent and a dark state, until they finally remain dark. This switching behavior is used for superresolution imaging. ${ }^{33}$ Importantly, the single molecules retain the contact sensitivity; that is, only those molecules confined by contact between the PS sphere and the glass substrate light up. ${ }^{34}$ Thus, by recording and statistically analyzing 4000 images, ${ }^{35}$ we obtain a superresolved image of the same PS-on-glass contact area (Figure 5). The resolution of this super-resolved image depends on the density of fluorescent molecules, which can vary locally. Nonetheless, it is clear from the edges of the contact patches that the resulting resolution is significantly better than the diffraction-limited microscopy image (Figure 5). We have quantified this improved resolution through the method described in ref 36 to be $152 \mathrm{~nm}$. To quantify the area of real contact in the super-resolved fluorescence image, we set an Otsu threshold, as in the regular microscopy images.

Thus, the area of contact measured by diffraction-limited microscopy is $194 \pm 15 \mu \mathrm{m}^{2}$, while the area of real contact measured through super-resolution imaging is $193 \pm 13 \mu \mathrm{m}^{2}$ : there is no significant difference between the results of the two techniques. We want to emphasize that indeed the main difference between the diffraction-limited and super-resolved images lies in the roughness of the edges of the contact patches and the appearance of many tiny contact patches. Both observations confirm that while high-frequency roughness does exist on the sphere and does lead to contact area structure that cannot be resolved by diffraction-limited microscopy, this fine structure does not strongly influence the total area of real contact. Persson's theory of the elastic contact of randomly rough solids has illustrated that lateral scales down to the atomic scale canand do-influence the area of real contact. ${ }^{37}$ However, in the elastoplastic version of the contact theory, a cutoff magnification emerges below which the area of real contact no longer depends on the resolution with which the interface is observed/analyzed. ${ }^{38}$ Based on our previous analysis, in which we investigated the area of real contact as a function of imaging resolution, ${ }^{23}$ and based on the present super-resolution observations, we therefore argue that in the PS-on-glass system, the cutoff length scale below which the structure in the area of real contact diminishes lies within the reach of optical imaging techniques. In other words; the contact patches observed using both imaging techniques in Figure 5 represent regions of the interface that are in full contact. Although earlier modeling work has indicated that such contact regions may contain atomic-scale structure, ${ }^{39}$ we argue that the large $(100 \mathrm{~nm})$ plastic deformation associated with the strain-hardening contact mechanics of the interfaces considered here ${ }^{22}$ precludes such effects. Our interpretation is further supported by fluorescence lifetime measurements, which will be discussed later in the paper. For the PS-on-glass contacts that are 
considered here, fluorescence intensity imaging therefore suffices, and furthermore, this technique is much faster than super-resolution imaging.

It follows from these results that the evolution of the friction force with waiting time cannot be accounted for solely by changes in the area of real contact; there must be an additional evolution of the quality of contact. To elucidate the nature of this evolution, we investigate in more detail the (diffraction-limited) fluorescence fingerprint of a contact between a rough PS sphere and a smooth glass substrate using FLIM (Figure 6). The contact is loaded with a normal force of $150 \mathrm{mN}$ and subsequently imaged both $20 \mathrm{~s}$ and 20 min after the normal force was applied.

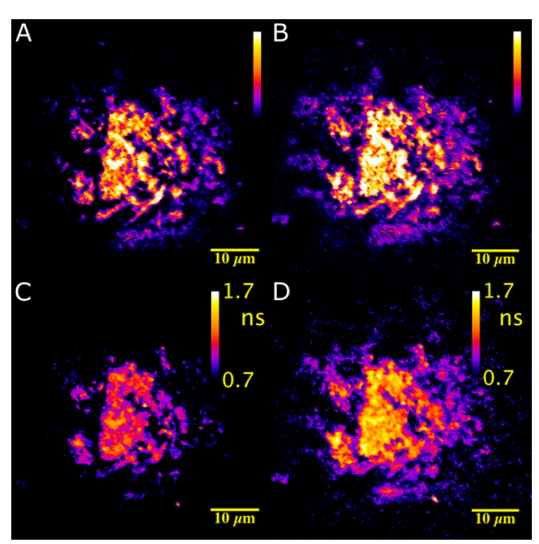

Figure 6. Fluorescence intensity images (FIM, top) and fluorescence lifetime imaging (FLIM, bottom) of the contact area between a PS sphere and a glass coverslip. Bright areas correspond to contact; elsewhere, the monolayer is immersed in formamide and remains dark. (A) FIM $20 \mathrm{~s}$ after contact formation. (B) FIM 20 min after contact formation. (C) FLIM $20 \mathrm{~s}$ after contact formation. (D) FLIM 20 min after contact formation.

During the 20 min waiting time, we observe a substantial increase in the fluorescence intensity recorded at the microscopic contacts; not only does the area of real contact grow but also the fluorescence intensity observed within this area of real contact increases. Fluorescence intensity is a concentration-dependent quantity; an increase in fluorescence intensity can in principle indicate both that more molecules are fluorescing (due to a larger area of contact) and that the degree of confinement (fluorescence) per molecule is increasing. Fluorescence lifetime, on the other hand, does not depend on how many molecules are excited within the diffraction-limited spot but indicates the degree to which the molecular rotors are confined; that is, how much free volume is available to perform twists along the intramolecular bonds: the more confined the molecules are, the slower the nonradiative decay and the longer the average lifetimes are. Importantly, we find that the measured increase in local fluorescence intensity is accompanied by an increase in fluorescence lifetime (Figure 6). We conclude that the fluorescence intensity does not increase because more molecules contribute to the fluorescence signal, that is, the contact area increases, but because the fluorescence intensity per molecule is going up as the contact ages. This enhanced fluorescence is a consequence of changes in the local environment of the probe molecules that inhibit intramolecular twists. As the contacts are fully resolved, the probe molecules signal that the density of their local environment increases as the contact ages under the influence of the normal force, thereby inhibiting intramolecular twists. Surprisingly, this compaction occurs while the average interfacial normal stress decreases because the normal force is constant and the contact area increases. We therefore interpret the observations in the context of an ageing glassy PS film, which becomes denser and mechanically stronger in time. An alternative interpretation of the data could be that chemical bonds are formed across the interface through a mechanism analogous to that demonstrated in recent atomic force microscopy (AFM) experiments. ${ }^{4,5,21}$ However, we observed that the transition from static to dynamic friction at the PS-on-glass interfaces considered here occurs over a sliding distance that is too large to be associated with the stretching of covalent bonds. ${ }^{23}$

It has been previously shown ${ }^{22,23}$ that strain hardening of the PS surface enables accommodation of an externally applied normal force; the PS surface becomes mechanically stronger as its strain hardens when it contacts a glass counter surface: the further the PS is strained, the larger the local normal stress it can withstand. Our results indicate that an analogous mechanism must apply for the shear strength of the PS; as the PS surface compacts in time-as indicated by the measured fluorescence lifetimes-under the influence of the normal force, the shear strength it can withstand before yielding increases: the friction per unit contact area goes up, as observed in the combined visualization/friction experiments.

The compaction-induced changes in the quality of contact are not exclusively observed at PS-on-glass interfaces. Previous experiments have demonstrated that the hardening behavior that defines the PSon-glass contact formation process also applies to PMMA-on-glass interfaces. ${ }^{22}$ In Figure 7, we show the ageing of the quality of contact for both PS and PMMA spheres in contact with smooth glass substrates. The measurements demonstrate that the PMMA contacts behave qualitatively similar to PS contacts: (i) the relative increase in friction with contact age is greater than that in contact area and (ii)

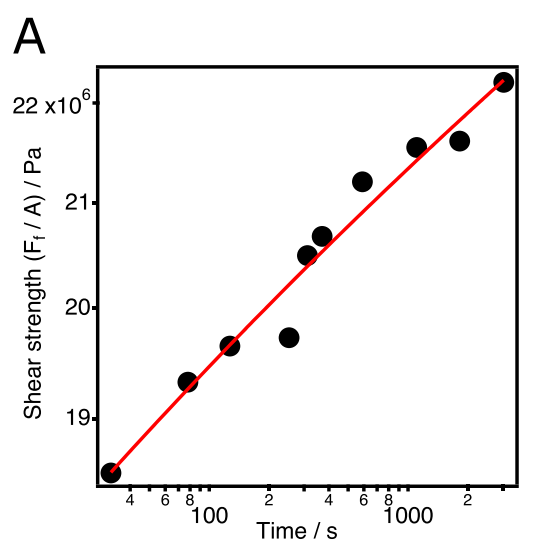

B

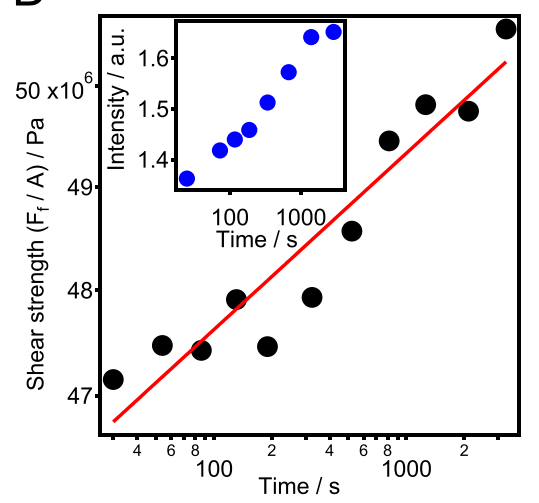

Figure 7. Contact shear strength as a function of contact age for different polymer beads on glass. The shear strength is defined as the static friction force, measured in slide-hold-slide experiments, divided by the area of real contact, measured by diffraction-limited fluorescence microscopy. (A) PS on glass. (B) PMMA on glass. The red lines serve as a guide to the eye. Friction and contact experiments were conducted as shown in Figure 2 (also see the Supporting Information). The inset in (B) shows the average fluorescence intensity within the area of real contact as a function of contact age, measured during a static ageing test in which the contact is only loaded in the normal direction. 
the fluorescence intensity observed within the area of real contact increases with contact age, signaling compaction of the interfacial polymers (Figure 7). Interestingly, the relative growth in shear strength for PMMA is roughly half that of PS. PS indeed can be compacted more easily than PMMA: ${ }^{22}$ by comparing strain-hardening contact calculations to fluorescence observations of the area of real contact between PMMA or PS spheres and glass, we previously showed that the strain-hardening parameter-defined as the increase in hardness with compaction-is $4 \mathrm{MPa} / \mathrm{nm}$ for PS and $7 \mathrm{MPa} / \mathrm{nm}$ for PMMA.

To independently link our fluorescence observations to the degree to which the interfacial PMMA is compacted, we also perform fluorescence lifetime measurements in which we either spin-coat a PMMA film on one of the DCDHF glass surfaces used in the contact experiments or dissolve DCDHF molecules in a PMMA matrix that is spin-coated onto a glass surface (i.e., not functionalized). For a polymer film-spin-coated on a glass surface-the layer closest to the interface is denser than the rest of the film because of the attractive van der Waals interaction between the polymer film and the glass surface. ${ }^{40}$ When such a film is spin-coated onto a functionalized coverslip, all DCDHF molecules are located within the dense region of the film, while the DCDHF molecules are present everywhere in the film if they are dissolved in the PMMA. We indeed observe that for the functionalized coverslips, the densification at the interface leads to an increased fluorescence lifetime- and thus fluorescence intensity-with respect to that measured when the DCDHF molecules are placed in bulk PMMA (Figure 8). Moreover, we find

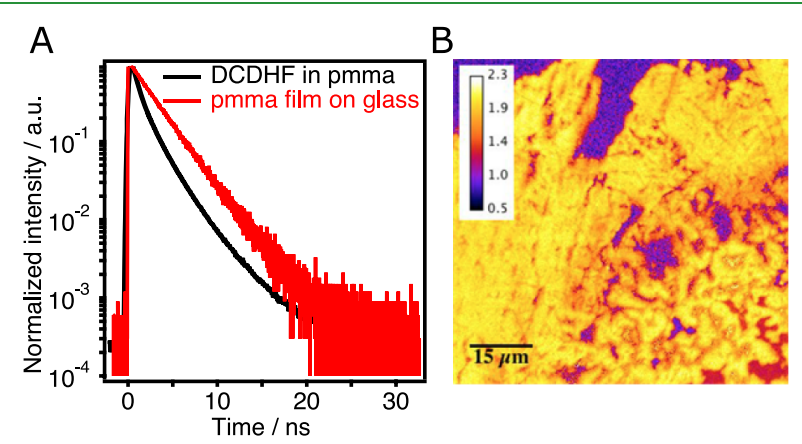

Figure 8. Time-correlated single photon counting (TCSPC) traces of the monolayer of DCDHF on glass with the PMMA film spin-coated on top of it (A, red curve, decay time $2.7 \mathrm{~ns}$ ) and DCDHF dispersed in the spin-coated PMMA film (A, black curve, $2.2 \mathrm{~ns}$ ). Each curve was obtained by averaging 20 TCSPC decays recorded at different locations at the interface. (B) FLIM (lifetime in ns) of the monolayer of DCDHF in contact with a PMMA sphere after 20 min of ageing.

that the fluorescence lifetime measured at mechanical PMMA/glass contacts is still lower than that of the spin-coated film. The aged contacts therefore do not yet reach the density limit of dense polymer films (Figure 8).

\section{CONCLUSIONS}

We show that at polymer-on-glass interfaces, the static friction force increases linearly with the logarithm of the contact time. To understand which role the area of real contact plays in this frictional ageing, we visualize the polymer-on-glass interface using confinement-sensitive fluorescent probe molecules that are immobilized on the glass surface. We critically evaluated our imaging techniques and conclude that diffraction-limited fluorescence microscopy can sufficiently resolve the area of real contact for the strain-hardening interfaces that were studied. While we do observe substantial growth of the area of real contact in time, this growth is not sufficiently strong to explain the evolution of the friction force with waiting time: the friction increases faster than the area of real contact. We find that within the area of real contact, the fluorescence lifetime increases as the contact ages. The fluorescence lifetime is insensitive to changes in the area of real contact but reflects the density of the direct environment of the probe molecules. We therefore conclude that the increase of static friction with contact age is caused by a combination of contact area and contact "quality" growth. The quality of the contacts is the shear stress they can withstand before yielding; as the polymer glass at the interface ages, it becomes denser and mechanically stronger. This time- and stress-dependent hardening behavior is expected to be strongly temperature-dependent; thus, temperature is an interesting parameter to probe in future experiments. Understanding the nature of static friction is of paramount importance to many applications in which friction plays a crucial role and plastics are widely used, that is, bearings, boat propulsion systems and the aviation industry.

\section{ASSOCIATED CONTENT}

\section{Supporting Information}

The Supporting Information is available free of charge at https://pubs.acs.org/doi/10.1021/acsami.9b19125.

Methods and materials: coverslip functionalization; friction/microscopy setup details; ball preparation; and additional friction measurements (PDF)

\section{AUTHOR INFORMATION}

\section{Corresponding Author}

B. Weber - Van der Waals-Zeeman Institute, IoP, University of Amsterdam, 1098XH Amsterdam, The Netherlands; Advanced Research Center for Nanolithography (ARCNL), 1098 XG Amsterdam, Netherlands; 이이. orcid.org/0000-0003-47564666; Email: b.a.weber@uva.nl

\section{Authors}

D. Petrova - van 't Hoff Institute for Molecular Sciences, University of Amsterdam, 1098 XH Amsterdam, Netherlands; (1) orcid.org/0000-0002-6682-0760

D. K. Sharma - Department of Materials Science and Engineering, Tokyo Institute of Technology, 152-8552 Tokyo, Japan

M. Vacha - Department of Materials Science and Engineering, Tokyo Institute of Technology, 152-8552 Tokyo, Japan; (1) orcid.org/0000-0002-5729-9774

D. Bonn - Van der Waals-Zeeman Institute, IoP, University of Amsterdam, 1098XH Amsterdam, The Netherlands; (1) orcid.org/0000-0001-8925-1997

A. M. Brouwer - van 't Hoff Institute for Molecular Sciences, University of Amsterdam, 1098 XH Amsterdam, Netherlands; (1) orcid.org/0000-0002-1731-3869

Complete contact information is available at: https://pubs.acs.org/10.1021/acsami.9b19125

\section{Notes}

The authors declare no competing financial interest.

\section{ACKNOWLEDGMENTS}

D.P. acknowledges funding from the Dutch Research Council NWO, ECHO Chemistry in Relation to Physics and Material Sciences 2013 CW, grant number 712.014.006. B.W. acknowledges funding from the Netherlands Organization for Scientific Research (NWO) VENI grant no. VI.Veni.192.177. This work 
is part of the FOM-Programme Fundamental Aspects of Friction and financed by FOM/NWO.

\section{REFERENCES}

(1) Dieterich, J. H.; Kilgore, B. D. Direct Observation of Frictional Contacts: New Insights for State-Dependent Properties. Pure Appl. Geophys. 1994, 143, 283-302.

(2) Heslot, F.; Baumberger, T.; Perrin, B.; Caroli, B.; Caroli, C. Creep, Stick-Slip, and Dry-Friction Dynamics: Experiments and a Heuristic Model. Phys. Rev. E: Stat. Phys., Plasmas, Fluids, Relat. Interdiscip. Top. 1994, 49, 4973-4988.

(3) Dillavou, S.; Rubinstein, S. M. Nonmonotonic Aging and Memory in a Frictional Interface. Phys. Rev. Lett. 2018, 120, 224101.

(4) Li, Q.; Tullis, T. E.; Goldsby, D.; Carpick, R. W. Frictional Ageing from Interfacial Bonding and the Origins of Rate and State Friction. Nature 2011, 480, 233-236.

(5) Tian, K.; Goldsby, D. L.; Carpick, R. W. Rate and State Friction Relation for Nanoscale Contacts: Thermally Activated PrandtlTomlinson Model with Chemical Aging. Phys. Rev. Lett. 2018, 120, 186101.

(6) Ben-David, O.; Rubinstein, S. M.; Fineberg, J. Slip-Stick and the Evolution of Frictional Strength. Nature 2010, 463, 76-79.

(7) Blass, J.; Bozna, B.; Albrecht, M.; Wenz, G.; Bennewitz, R. Molecular Kinetics and Cooperative Effects in Friction and Adhesion of Fast Reversible Bonds. Phys. Chem. Chem. Phys. 2019, 21, 1717017175 .

(8) Scholz, C. H. Earthquakes and Friction Laws. Nature 1998, 391, 37-42.

(9) Lee, D. W.; Banquy, X.; Israelachvili, J. N. Stick-Slip Friction and Wear of Articular Joints. Proc. Natl. Acad. Sci. U.S.A. 2013, 110, E567-E574.

(10) Hoffmann, N. P.; Gaul, L. Friction Induced Vibrations of Brakes: Research Fields and Activities. SAE Technical Paper Series, 2010; Vol. 1, p 724.

(11) Zhu, X.; Tang, L.; Yang, Q. A Literature Review of Approaches for Stick-Slip Vibration Suppression in Oilwell Drillstring. Adv. Mech. Eng. 2014, 6, 967952.

(12) Dong, C.; Shi, L.; Li, L.; Bai, X.; Yuan, C.; Tian, Y. Stick-Slip Behaviours of Water Lubrication Polymer Materials under Low Speed Conditions. Tribol. Int. 2017, 106, 55-61.

(13) van Bruggen, E.; van der Linden, E.; Habibi, M. Tailoring Relaxation Dynamics and Mechanical Memory of Crumpled Materials by Friction and Ductility. Soft Matter 2019, 15, 1633.

(14) Lahini, Y.; Gottesman, O.; Amir, A.; Rubinstein, S. M. Nonmonotonic Ageing and Memory Retention in Disordered Mechanical Systems. Phys. Rev. Lett. 2017, 118, 085501.

(15) Bowden, F.; Tabor, D. The Friction and Lubrication of Solids; Clarendon Press, 1954.

(16) Sakuma, H.; Kawai, K.; Katayama, I.; Suehara, S. What Is the Origin of Macroscopic Friction? Sci. Adv. 2018, 4, No. eaav2268.

(17) Lechthaler, B.; Ochs, G.; Mücklich, F.; Dienwiebel, M. Evolution of the True Contact Area of Laser Textured Tungsten Under Dry Sliding Conditions. Front. Mech. Eng. 2019, 5, 3.

(18) Sahli, R.; Pallares, G.; Papangelo, A.; Ciavarella, M.; Ducottet, C.; Ponthus, N.; Scheibert, J. Shear-Induced Anisotropy in Rough Elastomer Contact. Phys. Rev. Lett. 2019, 122, 214301.

(19) Sahli, R.; Pallares, G.; Ducottet, C.; Ben Ali, I. E.; Al Akhrass, S.; Guibert, M.; Scheibert, J. Evolution of real contact area under shear and the value of static friction of soft materials. Proc. Natl. Acad. Sci. U.S.A. 2018, 115, 471-476.

(20) Bureau, L.; Baumberger, T.; Caroli, C. Rheological aging and rejuvenation in solid friction contacts. Eur. Phys. J. E 2002, 8, 331337.

(21) Vorholzer, M.; Vilhena, J. G.; Perez, R.; Gnecco, E.; Dietzel, D.; Schirmeisen, A. Temperature Activates Contact Aging in Silica Nanocontacts. Phys. Rev. X 2019, 9, 041045.

(22) Weber, B.; Suhina, T.; Junge, T.; Pastewka, L.; Brouwer, A. M.; Bonn, D. Molecular Probes Reveal Deviations from Amontons' Law in Multi-Asperity Frictional Contacts. Nat. Commun. 2018, 9, 888.
(23) Weber, B.; Suhina, T.; Brouwer, A. M.; Bonn, D. Frictional Weakening of Slip Interfaces. Sci. Adv. 2019, 5, No. eaav7603.

(24) Hohlbein, J.; Gryte, K.; Heilemann, M.; Kapanidis, A. N. Surfing on a New Wave of Single-Molecule Fluorescence Methods. Phys. Biol. 2010, 7, 031001.

(25) Thorley, J. A.; Pike, J.; Rappoport, J. Z. Super-Resolution Microscopy; Elsevier Inc., 2014.

(26) Müller, M. Confocal Fluorescence Microscopy, 2nd ed.; SPIE: Bellingham, 2006.

(27) Petrova, D.; Weber, B.; Allain, C.; Audebert, P.; Venner, C. H.; Brouwer, A. M.; Bonn, D. Fluorescence microscopy visualization of the roughness-induced transition between lubrication regimes. Sci. Adv. 2019, 5, No. eaaw4761.

(28) Suhina, T.; Weber, B.; Carpentier, C. E.; Lorincz, K.; Schall, P.; Bonn, D.; Brouwer, A. M. Fluorescence Microscopy Visualization of Contacts Between Objects. Angew. Chem. Int. Ed. 2015, 54, 36883691.

(29) Suhina, T.; Amirjalayer, S.; Mennucci, B.; Woutersen, S.; Hilbers, M.; Bonn, D.; Brouwer, A. M. Excited-State Decay Pathways of Molecular Rotors: Twisted Intermediate or Conical Intersection? J. Phys. Chem. Lett. 2016, 7, 4285-4290.

(30) Rabinowicz, E. The Intrinsic Variables Affecting the Stick-Slip Process. Proc. Phys. Soc. 1958, 71, 668-675.

(31) Li, S.; Li, Q.; Carpick, R. W.; Gumbsch, P.; Liu, X. Z.; Ding, X.; Sun, J.; Li, J. The Evolving Quality of Frictional Contact with Graphene. Nature 2016, 539, 541-545.

(32) Gujrati, A.; Khanal, S. R.; Pastewka, L.; Jacobs, T. D. B. Combining TEM, AFM, and Profilometry for Quantitative Topography Characterization Across All Scales. ACS Appl. Mater. Interfaces 2018, 10, 29169-29178.

(33) Dempsey, G. T.; Vaughan, J. C.; Chen, K. H.; Bates, M. Evaluation of Fluorophores for Optimal Performance in LocalizationBased Super-Resolution Imaging. Nat. Methods 2012, 8, 1027-1036.

(34) Petrova, D.; Kumar, S. D.; Vacha, M.; Bonn, D.; Brouwer, A. M. Super-Resolution Imaging of Contacts between Objects. In preparation, 2020.

(35) Ovesný, M.; Kř́žzek, P.; Borkovec, J.; Švindrych, Z.; Hagen, G. M. ThunderSTORM: A Comprehensive ImageJ Plug-in for PALM and STORM Data Analysis and Super-Resolution Imaging. Bioinformatics 2014, 30, 2389-2390.

(36) Nieuwenhuizen, R. P. J.; Lidke, K. A.; Bates, M.; Puig, D. L.; Grünwald, D.; Stallinga, S.; Rieger, B. Measuring image resolution in optical nanoscopy. Nat. Methods 2013, 10, 557-562.

(37) Persson, B. N. J. Theory of rubber friction and contact mechanics. J. Chem. Phys. 2001, 115, 3840-3861.

(38) Persson, B. N. J. Elastoplastic Contact between Randomly Rough Surfaces. Phys. Rev. Lett. 2001, 87, 116101.

(39) Luan, B.; Robbins, M. O. The breakdown of continuum models for mechanical contacts. Nature 2005, 435, 929-932.

(40) Forrest, J. A.; Dalnoki-Veress, K.; Stevens, J. R.; Dutcher, J. R. Effect of Free Surfaces on the Glass Transition Temperature of Thin Polymer Films. Phys. Rev. Lett. 1996, 77, 2002-2005. 